\title{
A Revised Review on Opportunistic Routing Protocol
}

\author{
Shruti Pandey ${ }^{1}$, Mr. Ashutosh Rastogi ${ }^{2}$ \\ M.Tech $2^{\text {nd }}$ year, Wireless Communication and Sensor Network, Dept of Electronics and Communication, BBD University ${ }^{1}$ \\ Associate Professor, Department of Electronics and Communication, BBD University ${ }^{2}$
}

\begin{abstract}
Opportunistic routing is a new archetype in routing for wireless sensor network, designate the node closest to the target node to route the data. Opportunistic routing has recently fascinated much amenity as it is considered a promising proclivity for enhancing the performance, throughput and fidelity of wireless ad hoc sensor networks. It takes prevalence of the broadcast nature of Wireless sensor network for augmentation of transmission fidelity and network throughput. In this paper, we elucidate the foundation of opportunistic routing and the different regions where it is claimed to be better performer, then we discuss some of the popular routing protocols along with their metrics and contrasting drawbacks. Finally we conclude our paper with the existing issues and their possible remedies.
\end{abstract}

Keywords: WSN, Opportunistic Routing.

\section{INTRODUCTION}

Wireless Sensor networks (WSNs) are the network of spatially disseminates sensors which congregate information from the phenomenal world. It is used for audit environmental factors like temperature, pressure and moisture etc. and transmits the information or data to designated nodes [1]. WSN has been proven propitious in number of relevance such as traffic surveillance, military application, weather forecasting, landslide detection, fire detection etc.

Routing is gargantuan task in terms of wireless sensor network.Conspiring a routing protocol for wireless sensor network is different from conspiring it for the traditional networks. In case of the WSN, there is a strict energy saving requirement and there is issue of the increasing network lifetime. The main function of routing is the route selection and data forwarding. The route selection encompasses selecting the best route between two nodes. The data transmission is done by recruiting the next node or hop to forth data.

The packet propulsion in the traditional routing approaches for multi-hop wireless networks is done by nomating the node proactively at the sender side before transportal. A traditional multi-hop routing strategy abolishthe broadcasting nature of the wireless networks by using Automatic Repeat Request [ARQ] or Forward Error Control [FEC] Data link techniques [2]. The main infirmity of this strategy is that it simply implements main operations and principles congenital from bequest routing solutions that were initially apprehend for wired networks.

Indeed, traditional wireless routing protocols do not adapt well to the dynamic wireless environment variations. Hence, these protocols trigger excessive link-level retransmissions, unthriftiness of network resources, and may even lead to system cataclysm [3].

The adventconfabulate in this paper uses the broadcasting nature of wireless network for packet forwarding. This advent is named as "Opportunistic Routing (OR)". The pivotal idea behind OR is to adopt the broadcasting nature of the wireless network such that transmission from one node can be discovered by multiple nodes. In lieu of choosing the next forwarder node ahead of time, the OR chooses the next node dynamically at the time of transmission. The forwarding is done by node closest to the destination. It has been shown that OR gives better performance than traditional routing. The key task of the OR is to select the forwarder set and prioritize the nodes in the set [2].

Opportunistic routing (OR), also labeled asany path routing, has come forth a recent routing technology for wireless networks to take aid of the broadcast nature. By dynamically culling from multiple routes, OR can ameliorate link reliability and overall system throughput. 


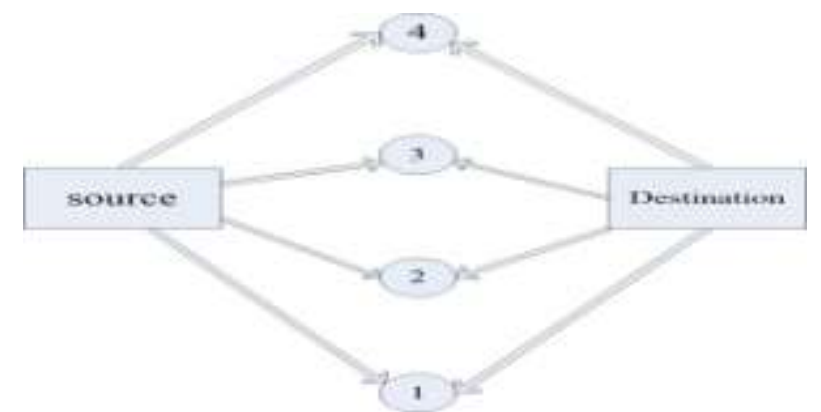

Fig.1: Illustration in which each source node has multiple intermediate nodes along with packet delivery for data transmission to the destination node.

Here the source node has four intermediate nodes with packet delivery. Each intermediate node has packet delivery probability of $85 \%$ to the destination. Traditional routing will select only one intermediate node for data forwarding, on the other hand OR will consider all these nodes for data forwarding. Thus, OR proves to be more efficient and reliable than traditional routing [1].

Opportunistic routing exploits accretion of same packet at multiple nodes in order to amend the network performance correlated to legacy routing. Indeed, by dynamically choosing the forwarder from set of multiple receivers, opportunistic routing can inevitably reduce number of packet retransmissions caused by the link failures. Thus, robustness is accommodated by opportunistic routing at a much lower cost (retransmission cost) in comparison to traditional routing [2]. This main asset, along with other ones, has emboldened the adoption of opportunistic routing in wide range of recent applications extended from emergency evacuation and recovery industrial sites interconnection, Internet provision in rural areas to building construction automation and critical infrastructure (water supply, electricity distribution, bridges and railroads, gas/oil pipelines) protection.

Currently, research on OR is still under development and botherations, such as channel assignment, routing and coordination, are not well considered. This paper studies and analyzes some existing OR schemes and explores possible issues. It begins with an introduction of primitive OR concepts, pursued by the differences between OR and other routing approaches. This paper distillate common solutions or improvements to these components from current OR schemes and portray them in detail. After which, several current OR protocols are introduced.

\section{A. ROUTING PARADIGM EVOLUTION}

In this section, we provide a brief audit of the routingcriterion evolution from its inception to its current state.

The need for routing standards first arose with emergence of the enterprise networks. The first two main approaches have been developed in this regard, namely Distance Vector Routing (DVR) [21] and Link State Routing (LSR) [22].

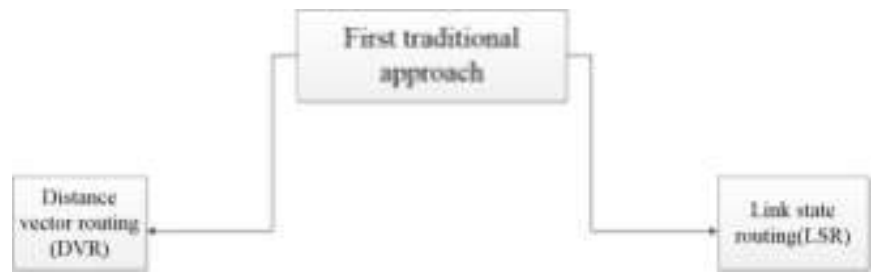

In the first approach (DVR),in this radically, each node systematically exchanges its distance vector information with its neighbors in order to arbitrate the next hop node from itself to each destination. The chief asset of this approach is that it only desire local information for the path computation. Despite its ease, in many cases DVR may agonize from slow convergence which leads to the other problems like routing loops. Therefore, this approach fits better for small local area wired networks, where the slow convergence is less likely to happen. The most commonly used DVR-based protocol is the RIP [21].

LSR, on the other hand, needs a global knowledge of network in order to arbitrate the best routing paths. LSR avoids routing loops to prejudice of higher computation complication and higher storage overhead. The most commonly used LSR-based protocol in wired networks is the OSPF [22]. 
Later, with the proliferation of Internet, the need to optimize these routing approaches has elevated. In this regard, a new technique that advances routing scalability has been developed. This technique is known as Hierarchical Routing [23]. The main attribute of the Hierarchical Routing is that it lies on apportion of the underlying network into altered domains in order to decrease the routing complexity in a large networks like Internet. Few years later, gratitude to its flexibility, low cost and ease of deployment, the wireless networks have started to replace wired networks whether in small areas (such as Wi-Fi-based WLANs, wireless Ad Hoc networks) or ample geographic regions (Wireless Mesh Networks). This fast conversion has incited research community and industry to develop new routing protocols that are bespoke to the unreliable nature of such networks. Among these protocols, we mention OLSR[24], AODV [25] and DSR[26]. These protocols use the clone path computation approaches designed for the wired networks (i.e. DVR and LSR) with some variations. For instance, OLSR is proactive link state routing protocol, designed for the routing in wireless LANs. In this protocol, static routes to all the destinations are pre-computed and maintained in routing tables at all times, whether they are essential or not. AODV, on the auxiliary, is a reactive distance vector routing protocol, which computes the routes to destinations only when they are needed or requested. DSR also comply on a demand source routing [23]. These routes are computed by a source node and piggybacked in packet header. In addition to these protocols, Hierarchical Routing protocols have been developed for the large wireless sensor networks. Similar to Internet case, these protocols lies on dispersing the network into different clusters in order to enable data aggregation, thereby inevitably contracting the network traffic load. Among these protocols, we mention LEACH, PEGASIS, TEEN, and APTEEN. Overall, these protocols are based on the traditional routing paradigms which were initially developed for the wired networks. Their main constraints are that they cannot be applied in the applications characterized by intermittent connectivity and anemic infrastructure like the disaster recovery applications. Moreover, the latter proliferation of heterogeneous wireless networks andtechnologies have created a need to better exploit this distinctpotential in order to boost the network capacity and toovercome the deficit of infrastructure in some areas. In the emergence ofthis new IT context, opportunistic routing drew great heed,within the last decade, benediction to its numerous advantages overtraditional routing [7]. The anatomy of the opportunistic routing concept was first architecture in ExOR [7] in 2005. The core of this protocol is toenhancethe performance of traditional routing schemes by capitalizing on the multiple transmission opportunities that the broadcast nature of wireless medium creates. The elemental operation of opportunistic routing is also refined in this protocol. Rather than (pre)selecting a specified relay node at every transmission, OR broadcasts data packet to the set of relay candidates. Then, relay candidates, who have profitably received the data packet, run a coordination protocol to select the optimum relay to forward the packet. In another word, OR is conceptually suppressed of these three steps:

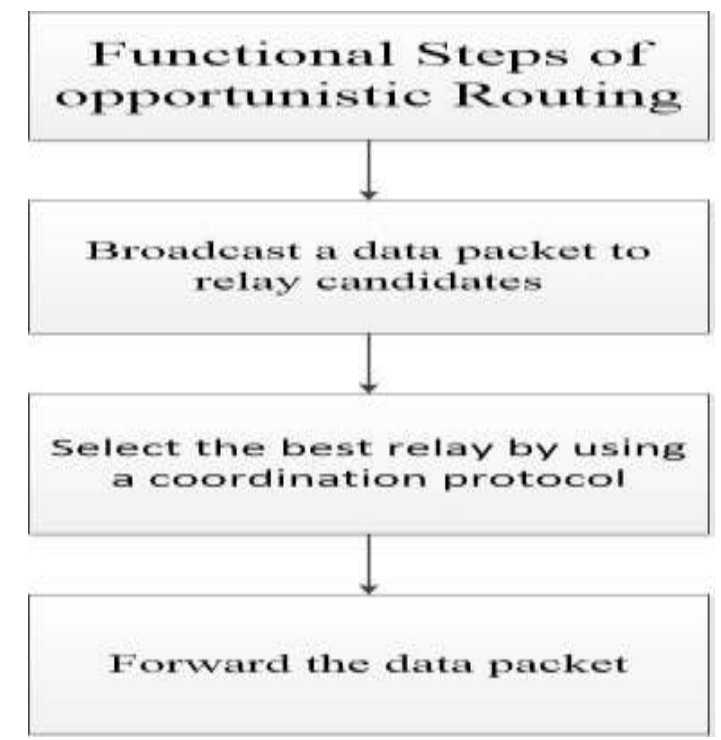

Fig.2: An illustration of steps of Opportunistic Routing

Notice here that the later hop relay is chosen after it has actually received packet, thereby contracting the number of packet retransmissions in comparison to traditional routing. An illustration of packet forwarding using opportunistic routing is shown in Fig. 3. In this source transmit the packet to the neighboring nodes and by the conceptual steps of opportunistic routing the packet is being transmitted to destination. 


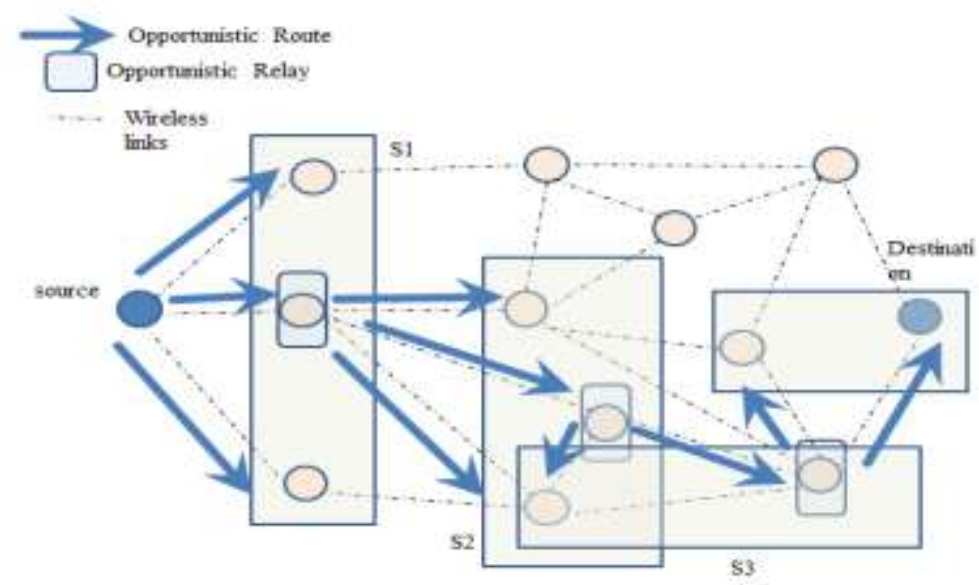

Fig.3: An illustration for packet forwarding in opportunistic routing.

B. Opportunistic Routing Advantage

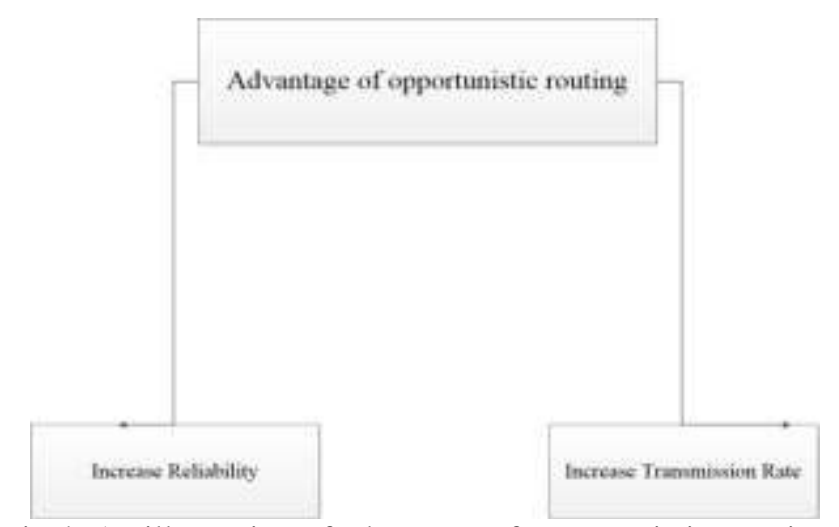

Fig.4: An illustration of advantage of opportunistic Routing.

a) Increase reliability:OR transmits the packet through any available link rather than one specified link. In other words, it acts like OR has additional substitute links and the probability of transmission collapse is decreased [5].

b) Increase transmission range: OR scrutinize all possible links, including ace quality short-ranged links and low quality long-ranged links, within one transmission; consequently, a transmission may precisely jump to the farthest relay which thrivingly receives the packet. Consequently, performance can be revised [5].

\section{Applications of Opportunistic Routing:}

Manyaspects make opportunistic routing the best routing surrogatein mobile and dynamic wireless networks as tothe main traditional routing approaches. For instance, one of themain constraints of LSR and DVR is the requirement to maintain networktopology information lastingly, whether it is local or global information.Comprehensively, traditional routing protocols suffer from high maintenance cost, reliance on static routes for the proactive protocols and high risk of using expired information for reactive ones.

Opportunistic routing, for the contrary, discards the need for expensive topology information allowance. It also reduces the count of packet retransmissions. As a matter of fact, wireless nodes do not have to worry about path or link breakages, considering only the candidate relays that have literally successfully received the packet compete in the forwarding process. Moreover, packets sent from the dupe source to the dupe destination may always take disparate paths depending on the opportunistic reception of the particular packets [19]. The majorassets of opportunistic routing over traditional routing are its flexibility and ease of adaptation to network dynamics. The characteristics benefit its adoption in many application synopses that cannot be handled by traditional routing. 


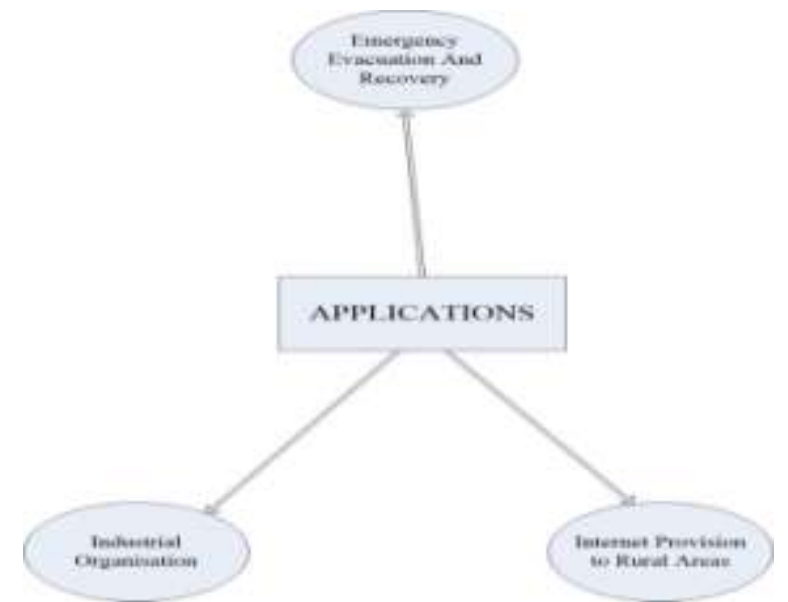

Fig.5: An illustration of Applications of Opportunistic Routing.

In the following headings, we give a brief analysis of some of these applications and explain the suitability of opportunistic routing to them [23]:

a. $\quad$ Emergency Evacuation and Recovery:

Gratitude to thefeatures offered by opportunistic routing, universal disaster recovery is being adorned, especially when finite or no infrastructure is available. Let us examine, for sample hurricane or earthquake affected areas. These disaster areas are generally characterized by a large demand or call attempt (from the disaster victims) on weakened network infrastructure .Opportunistic routing helps conquer these challenges by opportunistically selecting the best applicable links or nodes (mobile / fixed) to communicate with rescue and emergency personnel, thereby providing more resilient search and rescue operations.

b. Industrial Organization:

This application dwell in connecting industrial operations and sites just as oil and gas fields, mining and construction areas, which are complex to network because of their geography and their lossy breeding environments. Opportunistic routing best fits similar environments thanks to its adaptive nature. For specimen, in underground mine sites characterized by tremendous signal attenuation and frequent network disruptions, opportunistic routing grant field workers to always connect to the best communication unit in their circumferentialand have their calls or traffic loyally transferred to the identicalcentral safety unit.

c. Internet Provision to Rural Areas:

Given the absence of infrastructure in rural areas, opportunistic routing provides anominal alternative to Internet provision. In fact, by opportunistically using the obtainable wireless devices as relays, opportunistic routing endeavor a better Internet coverage, thereby helping to link the digital gap.

Due to the sporadic nature of the communications eventuate in these applications (especially application (a) and (c)), as well as the scarcity of infrastructure, traditional routing could not be used. In fact, traditional routing cannot acclimate to the frequent changes in these networks as averse to opportunistic routing which bid more flexibility and robustness.

\section{ANATOMY OF OPPORTUNISTIC ROUTING IN WIRELESS MULTIHOP NETWORKS}

The opportunistic routing prototype first appeared in 2005, with the design of first opportunistic routing scheme, ExOR [7], which was performed on the Roof Net testbedat the Massachusetts Institute of Technology.

The target of this protocol is to reform the performance of traditional routing schemes by exploiting multiple transmission opportunities that broadcast nature of wireless medium creates. Proceeding to that, utmost of the routing schemes proposed for generic Ad hoc networks (such as DSR, AODV and OLSR) a chosen shortest path between a source and a destination pair, and forward specific packet through a predetermined continuity of intermediate nodes. In the last decade, opportunistic routing drew enormous attention within the research community credit to its attractive applications as well as its several advantages over traditional routing. 


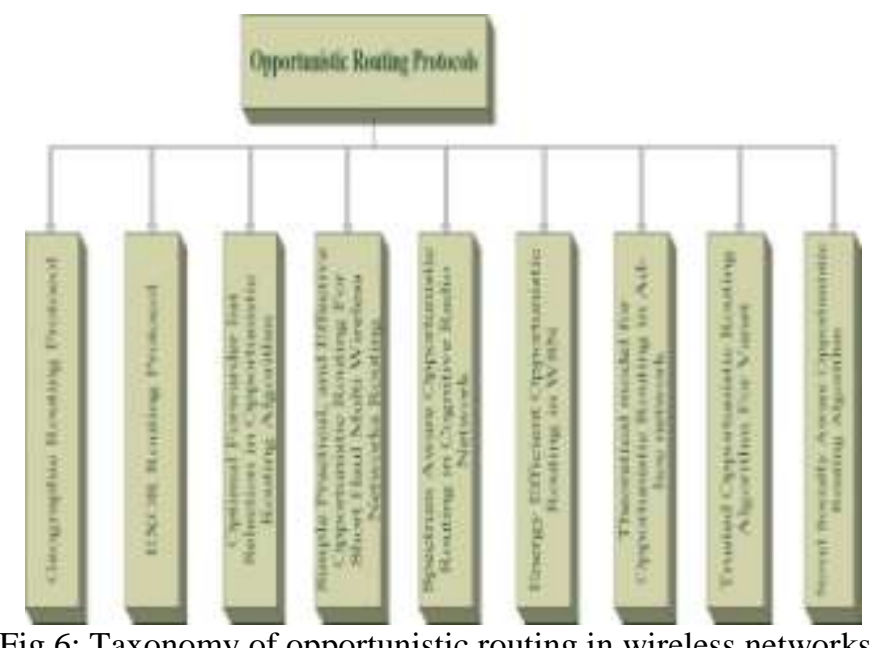

a) Geographic Random Forwarding (GeRaF) for Ad Hoc and Sensor Networks: Multihop Performance [6] In this paper, scheme termed Geographic Random Forwarding, which is based on geographic routing. In wireless network the relay node is not known by the sender but is assured after the transmission. It adoptsthe telecasting nature of the wireless network. Since the topologies are randomly bartered, the sender node does notknow which of its neighboring node will act as a relay node. Hence, to deal with conflict at the receiver end,author has proposed the above scheme. The elemental idea of the paper is as follows: The sender node easefully broadcaststhe packet along with its own location and destination location. The entire listening node in neighbor will receivethe packet and based on the peculiar distance from destination, they prioritize themselves to act as relay node. Therelayed packet is then appointed to a broadcasting address which also contains the transmitter and final destinationlocation thus providing a geographic route without maintaining routing table.

Thus this describes the forwarding approach based on geographic location and randomselection of relay node through contention at the receiver side. The analysis of multihop performance isdone in terms of the number of hops to reach to the destination as a function of distance and the number of nodes inthe neighbor nodes.

b) ExOR: Opportunistic Multi-Hop Routing for Wireless Networks [7]

This is the first most basic protocol which approximately implemented the Opportunistic Routing in the wireless networks. ExOR uses batches to send packets. The source node congregates the packets which are intended to dupe destination and gangs them into a batch. Each batch has its own Batch ID. The source node selects the BatchID and the forwarder list prioritized based on the ETX metrics,diminished the distance of node from target node higherthe priority. Only the nodes having higher priority are embodied in the forwarder list. Each node in the forwarder listcultivates a local batch map. The node computes the packet into the packet buffer for analogous batch. The noderelates the entry for each batch map in the packet with comparable entry in the local batch map and if thehigher priority entry is recognized, it reinstates the entry in the local batch map. ExOR implements anticipatedtransmission of packets to ensure that only one node sends the packet at one time.

c) Optimal Forwarder List Selection in Opportunistic Routing [9]

In this paper, MTS algorithm has been proposed for selecting forwarding list which lessen the expected transmission rate under the ideal ACK condition. The presumption made here is that low priority nodes can always herebroadcast of the high priority node thus there will be no clone transmission of packets. Under this hypothesis the Minimum Transmission Scheme algorithm is been proposed, which computes the optimal forwarding list. While applying this algorithm in ExOR instead of the ETX, the MTS based ExOR gives lesser transmissions than that of the ETX based ExOR. Thus the throughput of the MTS based ExOR is prominent than that of the ETX based ExOR. However in certain cases when the perfect ACK condition is not satisfied, the ETX based ExOR performs well than the MTS based ExOR.

d) Simple, Practical, and Effective Opportunistic Routing for Short-Haul Multi-Hop Wireless Networks [11] Here the effective opportunistic routing scheme for short haul multi-hop wireless networks has been proposed. This modified Opportunistic routing algorithm contrivance the scheme of sending the ACK after receiving packet. 


\section{International Journal of Advanced Research in Computer and Communication Engineering ISO 3297:2007 Certified \\ Vol. 6, Issue 4, April 2017}

In this algorithm solitary destination can opportunistically receive the packet by overhearing the transmission of nodes in traditional networks. After destination node receives the packet from priori node it sends ACK to all other nodes in path. The node will only retransmit the packet in case it did not receive the packet from either destination or next node in path. Hence, the destination node can easily discard any clone packets. Thus this algorithm lowers the packet duplication rate. Also it enhances the throughput than that of the other opportunistic algorithms. It is ease and can be integrated with other Opportunistic algorithm.

e) Spectrum Aware Opportunistic Routing in Cognitive Radio Networks [12]

Shih-Chun Lin and Kwang-Cheng Chen introduce the SAOR i.e. Spectrum Aware Opportunistic Routingfor Cognitive Radio Network (CRN). The algorithm proposed by the authors avail the optimal link transmission (OLT) as a cost cadent for prioritizing the nodes in forwarded list. The OLT metric is contemplated in the delayaspect. Two more metrics namely optimal path metric and node metrics further amplify the count of hops in thepath and the delay prominence within each path respectively to the destination. Due these metrics SAOR gives QoS guarantees like better throughput and upgraded end to end delay performances than traditional routing algorithms for $\mathrm{CN}$.

f) Energy-Efficient Opportunistic Routing in Wireless Sensor Networks [33]

This proposes the strategy to choose the forwarding list using cost cadent of minimum energy depletionwhile broadcasting in wireless sensor network. Energy Efficient Opportunistic Routing (EEOR) enumerates the expected cost for each node to forward data and then elects the forwarding list. The base of selecting forwarding list is that conventional cost of the node to be summoned must be less than prefix forwarding list so that the absolute expected cost will be minimal for new forwarding list. The forecasted cost updating of each node is done by the algorithm collateral to the Bellman Ford algorithm. EEOR avails less time than that of ExOR for twain transmission and receiving data. Due to costmetrics cogitated in EEOR, the average size of forwarding list of the EEOR is much less than that ofExOR. In case of entire energy consumption, EEOR performs more appropriate than EXOR. In comparison of both the protocols for packet loss rate and end to end delay EEOR performance is more valuable than ExOR.

g) A theoretical model for opportunistic routing in ad hoc networks [34]

The scheme proposes the general framework to facsimile opportunistic routing. It availsdelivery ratios and the priority order among nodes to select the adjacent hop for packet forwarding. It targets on giving a closed form expression for average transmission numeral. This prototype helps to analyze the performance parameters equivalent to packet dropping rate, packet transmission number, end to end packet delay etc.

h) A Trusted Opportunistic Routing Algorithm for VANET [35]

The paper chronicles the trust mechanism for the security bug in the opportunistic routing algorithm.It describes the tradeoff among the cost metric and security factor. According to survey, severalauthorspropose the algorithm which account the degree of trust and amend the direct trust degree. The degree of trust is stationed on the direct observation of neighboring nodes while indirect trust degree is planted on the recommendation. By using particular factors implementation has been performed for the Trust Opportunity Forwarding Mechanism using cost effective forwarding list and has prioritize each and every node in list by its cost distance from destination. The TMCOR algorithms do justice to well in all three metrics viz. throughput, end to end packet delay and security gains. TMCOR ban malicious node to participate in network by judging them in premise of trust degree. Thus, reducing End to End packet delay and increasing security gains.

i) A Novel Socially-Aware Opportunistic Routing Algorithm in Mobile Social Networks [36]

The author has contemplated the use of OR for the MANETs using the cost metrics alike social relations andprofiles of the nodes. The proposed distributed protocol is Social Relation Opportunistic Routing (SROR) to compute best forwarding node in routing. The protocol mainly considers the social relations, mobility patterns and social profiles for Mobile Ad-hoc Networks (MANETs). For selection of the forwarding node in the routing SROR following three matching parameters are taken into account viz. social profile matching, social connectivity matching and social interaction. Hence when the node wants to send the packet, due to the algorithm there is high possibility that the best candidates sharing similar interest tend to meet again to forward the data. SROR gives high packet delivery rate and routing efficiency compared to other protocols for MANETs.

j) Opportunistic Routing Algorithm for Relay Node Selection in Wireless Sensor Networks [37]

This paper concerns about the energy savings concept in WSNs. It characterizes the algorithmwhich cynosure on minimizing the energy consumption of network. The author has proposed Energy Saving via Opportunistic Routing (ENS- 
OR). The algorithm implements concept of energy efficient node (EEN) which appears to be aimplicit relay node obtained by relay function on certain real nodes based on their surplus energy.

The forwarder list selection and prioritizing nodes in thatballot is carried out by ENS-OR algorithm measure the optimal hop distance to calculate the next hop node to forward data. The nodes in forwarding list prioritize themselves by their surplus energy and their distance from the EEN.

ENS-OR obtains improved network energy usage. Also it enhances the network lifetime by accomplishing higher residual energy of nodes in the network. The packet delivery rate of the ENS-OR is much higher than that of GeRaF.

\section{E. DEMUR AND AGAPE CONTRADICTIONS}

Despite the potential asset that OR can bring to the wireless networks, prevailing demur remain and more research is desired to augment OR. In this classification, we debate some of the demur faced by OR and the agape contradiction that are yet to be adobe [10].

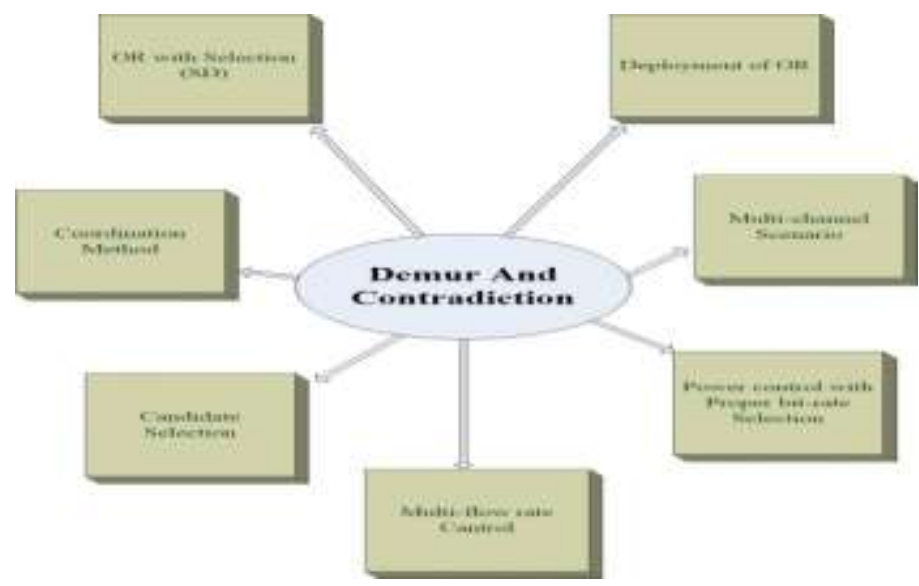

Fig.7: Demur and agape contradiction of opportunistic routing protocol.

i. Candidate selection:

While various candidate selection schemes have been advised, there is absence of integration of altered desirable features. Typically, filtering and ordering metrics are adapted separately such that the conclusion is not optimized. For example, filtering by dualisticprobability abolishes candidates that trigger dualistic transmissions after the supreme candidate order has been generated using the given metric. Although both approaches target for the same goal of boost performance, the output yield may not necessarily be the best as the later phase filtering can also adjust the optimized candidate order that has been earlier generated using the metric in the beginning phase. Consequently, to get an optimized candidate order, anunified approach taking all expected features, such as dualistic probability and virtual link strength, into consideration is highly commended.

ii. Power control with proper bit-rate selection :

Power control is a captious issue in the wireless networks. From preceding studies, a variable-range transmission approach sustained by power control can outperform an anchored-range transmission approach in condition of power savings and enhanced capacity [28]; besides, it has been advertised that performance improvements of more than $100 \%$ can be accomplished with power control over accustomed non-power-controlled 802.11 networks [29]. Hence , supporting OR by power-control is desirable.

iii. Multi-flow rate control:

As it has been announced that TCP congestion control is not applicable for multi-hop wireless networks, various solutions have been considered to cope with it. For OR, minor rate control solutions, such as, in SOAR and CCACK, have been advised. As these clarifications have been calculated mainly by simulations with few testbed experimentations, more thantheoretical studies on the rate control for OR are required.

iv. Multi-channel scenario :

Another popular advancement to improve the capacity of the wireless networks is multi-channel multi-interface approach. OR believe on overhearing; contrary to, the multi-channel advent improves capacity by compressing overhearing and the 
interference from neighbors. In other words, lesser candidates are accessible for OR in a multi-channel scenario [22]. Furthermore, Zubow, et al. [30] showed analogous simulation output that the inclusive throughput enhances proportionally to the increase in count of channels. OR has the boon to overcome deafness problem, where nodes cannot acquaint with each other because they accomplish on different channels. Though OR in multi-channel scenario is promising, the channel assignment approach for OR is still an open research problem.

v. Combination OR with Selection Diversity (SD) :

Though SD does not gain from overheard packets as OR, it is adventitious and beneficial to put them together. SD is shown to have great appulse on performance in slow fading channel conditions with multirate support [23].However, in fast fading channel case, the channel condition information of SD is antiquated and the performance of SD drops somewhat due to probing overhead. In contrary to, multiple backup links and without probing cost, OR surpass SD in fast changing channel conditions. Summarily, under multirate scenarios SD is suitable for slow fading channel conditions while OR is good for fast fading channel.

\section{vi. Deployment of OR:}

Numerical and simulation results have demonstrated the potential of OR, but the applicable network topology to be accepted for deployment has not been examined yet. The requirement for OR deployment can be discussed in two aspects:

A) Transitioning from current wireless mesh networks.

B) Building the new wireless mesh network

Due to the inheritance of OR, traditional deployment strategies may not actualize the best tradeoff between performance and cost (number of relays), i.e. cost-performance ratio for constructing the wireless mesh network. Besides, even in aoutlined and optimized wireless network for traditional routing, OR is still profitable as long as link quality is not absolute (i.e., varies over time due to changing environmental case) and multiple relays are accessible. Though existing wireless mesh networks can be comfortably modified to take benefits of OR by modernizing their firmware, the main demur is that those nodes are mostly immotile and therefore the network topologies may not be conveniently reorganized. As a result, there is a devoir need to determine if it is favorable to implement OR using the current network deployment.

\section{vii. Coordination method:}

1) Token-based coordination-

Token-based coordination is complimentary from duplicates and is admirable in scenarios such as grids. The token-passing path significantly credits its performance; however, the encounter of the path election has not been analyzed yet and solutions to choose the optimal token-passing path(s) are also inadequate.

2) Network coding coordination -

Though network coding coordination has the dormant to achieve zero overhead, coding time and redundant packets are the main contradictions. It has been shown that the integrated of network coding and OR can be implemented on low-end PCs, but it still cannot be deployed on current wireless networking devices. In others words, for actual wireless mesh networks upgrading from the traditional routing to OR, network coding coordination is not an option. Regarding the contradiction of redundant packets, the timing of coded packet generation is critical. Previous calculations based on offline prediction are inaccurate [13]; as a result, the effect of redundant packets may be even greater. The issue of when to generate coded packets certainly requires further analysis.

\section{CONCLUSION}

In this paper, we provide a comprehensive survey of opportunisticrouting protocols. Specifically, we first introduce themain factors and applications that lead to the development ofthe opportunistic routing paradigm. The evolution through which opportunistic routing came in major in wireless sensor network with its advantages. Then the application of opportunistic routing protocol, how is been useful in real world scenario. Then we briefly describe the protocols of opportunistic routing. Lastly, we presents the open issues and challenges yet to be solved of opportunistic routing.

\section{REFERENCES}

[1]. A Survey on Opportunistic Routing Protocols for Wireless Sensor Networks Payal Jadhav*, Prof. RachnaSatao.

[2]. XiaohuaXu, Student Member, IEEE, Xiang-Yang Li, Senior Member, IEEE, and Huadong Ma, Senior Member.” Energy-Efficient Opportunistic Routing in Wireless Sensor Networks"IEEE Transaction on Parallel and Distributed Systems, VOL. 22, NO. 11, Nov 2011. 
[3]. Haitao Liu and Baoxian Zhang, Chinese Academy of Sciences Hussein T. Mouftah, University of OttawaXiaojunShen, University of Missouri Kansas CityJian Ma, Nokia Research Center "Opportunistic Routing for Wireless Ad Hoc and Sensor Networks: Present and Future Directions"IEEE Transaction on communication magazine, VOL. 22, NO. 11, Dec 2009.

[4]. H. Liu, B. Zhang, H. Mouftah, X. Shen, and J. Ma, "Opportunistic routing for wireless ad hoc and sensor networks: Present and future directions," IEEE Commun. Mag., vol. 47, no. 12, pp. 103-109,Dec. 2009

[5]. Che-Jung Hsu a, Huey-Ing Liu b*, Winston K.G. Seah," Opportunistic Routing - A Review and the Challenges Ahead", IEEE Transaction on Parallel and Distributed Systems, VOL. 22, NO. 11, Nov 2014.

[6]. M. Zorzi and R.R. Rao, "Geographic Random Forwarding (GeRaF) for Ad Hoc and Sensor Networks:Energy and Latency Performance,"IEEE Trans. Mobile Computing, vol. 2, no. 4, Oct.-Dec. 2003.

[7]. S. Biswas and R. Morris, "ExOR: Opportunistic multi-hop routing for wireless networks," in Assoc. Comput. Mach. SIGCOMM Comput. Commun. Rev., 2005, vol. 35, no. 4, pp. 133-144.

[8]. D. De Couto, D. Aguayo, J. Bicket, and R. Morris, “A high-throughput path metric for multi-hop wireless routing In Proc. ACM/IEEE MobiCom, September 2003.

[9]. YanhuaLi , Wei Chen, and Zhi-Li Zhang," Optimal Forwarder List Selection in Opportunistic Routing”Mobile Ad hoc and Sensor Systems. IEEE 6th international conference.

[10]. X. Zhang and B. Li. Dice: "a game theoretic framework for wireless multipath network coding". In Proceedings of ACM MobiHoc, 2008.

[11]. Goo Yeon Lee and Zygmunt J. Haas,"Simple, Practical, and Effective Opportunistic Routing for Short-Haul Multi-Hop Wireless Networks", IEEE Transaction on Wireless Communication, VOL. 10, NO. 11,Nov 2011.

[12]. Shih-Chun Lin and Kwang-Cheng Chen"Spectrum Aware Opportunistic Routing in Cognitive Radio Networks". IEEE Globecom 2010 proceedings.

[13]. S. Diggavi, N. Al-Dhahir, A. Stamoulis, and A. Calderbank, "Great expectations: The value of spatial diversity in wireless networks," Proc.IEEE, vol. 92, no. 2, pp. 219-270, Feb. 2004.

[14]. X. Qin and R. Berry, "Exploiting multiuser diversity for medium access control in wireless networks," in Proc. IEEE Conf. INFOCOM, 2003, pp. $1084-1094$.

[15]. Gorbil and E. Gelenbe, "Resilient emergency evacuation using opportunistic communications," in Proc. 27th ISCIS, Oct. 2012,pp. 249-257.

[16]. Martin-Campillo, J. Crowcroft, E. Yoneki, and R. Marti, "Evaluating opportunistic networks in disaster scenarios," J. Netw. Comput. Appl., vol. 36, no. 2, pp. 870-880, Mar. 2013.

[17]. S. Yoon, S. Jang, Y. Kim, and S. Bahk, "Opportunistic routing for smart grid with power line communication access networks," IEEE Trans.Smart Grid, vol. 5, no. 1, pp. 1949-3053, Jan. 2014

[18]. Pentland, R. Fletcher, and A. Hasson, "DakNet: Rethinking connectivity in developing nations," IEEE Comput. Mag., vol. 37, no. 1, pp. 78- 83, Jan. 2004.

[19]. K. Zeng, W. Lou, H. Zhai, Capacity of opportunistic routing in multi-rate and multi-hop wireless networks, IEEE Transactions on Wireless Communications, 7 (2008) 5118-5128.

[20]. C.-P. Luk, W.-C. Lau, O.-C. Yue, An Analysis of Opportunistic Routing in Wireless Mesh Network, in: Proceedings of the IEEE International Conference on Communications (ICC), Beijing, China, 2008.

[21]. C. Hedrick, "Routing information protocol," Internet Requests for Comments, RFC Editor, RFC 1058, Jun. 1988

[22]. J. Moy, "OSPF version 2," Internet Requests for Comments, RFC Editor, RFC 2178, Jul. 1997

[23]. A Survey on Opportunistic Routing in Wireless Communication Networks Nessrine Chakchouk, Member, IEEEIEEE COMMUNICATION SURVEYS \& TUTORIALS, VOL. 17, NO. 4, FOURTH QUARTER 2015.

[24]. T. Clausen and P. Jaquet, "Optimized Link State Routing Protocol (OLSR)," Internet Requests for Comments, RFC Editor, RFC 3626 , Oct. 2003.

[25]. C. Perkins, E. Belding-Royer, and S. Das, "Ad Hoc On-Demand Distance Vector (AODV) routing," Internet Requests for Comments, RFC Editor, RFC 3561, Jul. 2003

[26]. D. Johnson, Y. Hu, and D. Maltz, "The Dynamic Source Routing Protocol (DSR) for mobile ad hoc networks for IPv4," Internet Requests for Comments, RFC Editor, RFC 4728, Feb. 2007.

[27]. N. Chakchouk, B. Hamdaoui, and M. Frikha, "WCDS-DCR: An energyefficient data-centric routing scheme for wireless sensor networks,"WirelessCommun. Mobile Comput. J., vol. 12, no. 2, pp. 195-205,Feb. 2012.

[28]. W. R. Heinzelman, A. Chandrakasan, and H. Balakrishnan, "Energyefficient communication protocol for wireless microsensor networks," in Proc. IEEE Int. Conf. Syst. Sci., Jan. 2000, pp. 1-10.

[29]. S. Lindsey and C. S. Raghavendra, "PEGASIS: Power efficient gathering in sensor information systems," in Proc. IEEE Aerosp. Conf., Mar. 2002, pp. $1125-1130$

[30]. A. Manjeshwar and D. P. Agrawal, "TEEN: A protocol for enhanced efficiency in wireless sensor networks," in Proc. Int. Workshop Parallel Distrib. Comput. Issues Wireless Netw. Mobile Comput., Apr. 2001, pp. 2009-2015.

[31]. A. Manjeshwar and D. P. Agrawal, "APTEEN: A hybrid protocol for efficient routing and comprehensive information retrieval in wireless sensor networks," in Proc. Int. Workshop Parallel Distrib. Comput.Issues Wireless Netw. Mobile Comput., Apr. 2002, pp. 1-8.

[32]. W. Zaumen and J. G.-L. Aceves, "Dynamics of distributed shortest-pathrouting algorithms," in Proc. Conf. Commun. Archit., Protocols Appl.SIGCOMM, Sep. 1991, pp. 31-42.

[33]. XiaohuaXu, Student Member, IEEE, Xiang-Yang Li, Senior Member, IEEE,andHuadong Ma, Senior Member." Energy-Efficient Opportunistic Routing in Wireless Sensor Networks"IEEE Transaction on Parallel and Distributed Systems, VOL. 22, NO. 11, Nov 2011.

[34]. Angela Sara Cacciapuoti, Marcello Caleffi and Luigi Paura "A theoretical model for opportunistic routing in ad hoc networks"Ultra Modern Telecommunications \& Workshops, 2009

[35]. JieZhingzhong, Huanchuanhe, XuLiya, Wang Bo, Chen Xi, Fan Xiying.”A Trusted Opportunistic nRouting Algorithm for Vanet" Third International Conference on Networking and Distributed Computing 2012.

[36]. Gary K. W. Wong, XiaohuaJia," A Novel Socially-Aware Opportunistic Routing Algorithm in Mobile Social Networks" International Conference on Computing, Networking and Communications, Wireless Ad Hoc and Sensor Networks Symposium 2013.

[37]. Juan Luo, Member, IEEE, Jinyu Hu, Di Wu, Member, IEEE, and Renfa Li, Senior Member, IEEE“'Opportunistic Routing Algorithm for Relay Node Selection in Wireless Sensor Networks" IEEETransactions on Industrial Informatics, VOL. 11, NO. 1, February 2015. 